Theoretical Physics, Astrophysics and Cosmology

Vol. 4, No 1, p. 6-9, v1, 19 March 2009 Online: TPAC: $3000-013$ v2, 18 September 2012
ISSN 1819-9135; ISSN 1991-3919 (e)

(C) 2009, 2012 CTPA. All rights reserved. DOI: $10.9751 /$ TPAC.3000-013

\title{
Gravitation as a Quantum Diffusion ${ }^{1}$
}

\author{
Zahid Zakir ${ }^{2}$
}

\begin{abstract}
Inhomogeneous Nelson's diffusion in flat spacetime with a tensor of diffusion can be described as a homogeneous one in a Riemannian manifold with this tensor of diffusion as a metric tensor. The influence of matter to the energy density of the stochastic background (vacuum) is considered. It is shown that gravitation can be represented as inhomogeneity of the quantum diffusion; the Einstein equations for the metrics can be derived as the equations for the corresponding tensor of diffusion.
\end{abstract}

PACS: 04.20.Cv, 03.65.Ta, 05.40.Jc, 04.62.+v

Key words: stochastic mechanics, tensor of diffusion, quantum fluctuations, gravitation, curvature

\section{Content}

Introduction 6

1. Sample particles on inhomogeneous stochastic background............................................. 7

2. The influence of matter to the stochastic background .................................................... 7

References 9

\section{Introduction}

In the paper [1] inhomogeneous conservative diffusion with a tensor of diffusion $v^{a b}(x, t)$ has been considered and the similarities in the geometric descriptions of gravitation and quantum fluctuations have been discussed. The proposed idea about the physical nature of gravitation is that gravitation is inhomogeneous Nelson's diffusion, i.e. a consequence of the quantum fluctuations. This treatment may be applied also to some gauge fields by means of the Kaluza-Klein mechanism.

In [1] only the behavior of a sample particle on inhomogeneous stochastic background has been described. In this paper the influence of the source to this stochastic background will be considered, and gravitation will be described fully in terms of the general quantum diffusion. Then the Einstein equations will be related with the corresponding diffusion equations.

Two basic phenomena of physics - the gravitation and quantum fluctuations both depend only on masses of objects and both have a geometric nature. The geometrical origin of gravitation is well known, while up to now Nelson's discovery of the stochastic geometry of spacetime [2] does not accepted as one of fundamental concepts of physics. The stochastic mechanics is a unique version of quantum mechanics

\footnotetext{
${ }^{1}$ The preprint of the paper has been presented in 1999 (revised 2003): Zakir Z. arXiv:gr-qc/9906079.

${ }^{2}$ Centre for Theoretical Physics and Astrophyics, Tashkent, Uzbekistan; zahidzakir@theor-phys.org
} 
here the quantum fluctuations are represented as manifestations of stochastic geometry of spacetime with a constant diffusion coefficient $v_{0}=\hbar / 2 m$ (see also the review [3]).

It is natural to consider a more general case of spacetimes with inhomogeneous diffusion by a tensor of diffusion $v^{a b}(x, t)$. In the paper it will be shown that the stochastic structure of the spacetime with such tensor of diffusion induces a nontrivial metric $g^{a b}(x, t)$ and a curvature. This means that gravitation can be treated as a quantum diffusional effect.

The fact that the quantum fluctuations and gravitation are not independent phenomena, leads to an interesting solution of the problem of the gravitational energy. As it is well known, the energy density of the instantaneous Newtonian interaction, as attractive one, is negative defined and in the diffusional treatment of gravity this property can be naturally explained.

\section{Sample particles on inhomogeneous stochastic background}

At inhomogeneous diffusion, considered in [1], the mean acceleration $E\left[a^{i}(x, t)\right]$ does not contain terms with the osmotic velocity $u^{i}$ and for the diffusion of a free particle we have:

$$
E\left[\frac{\partial v_{i}}{\partial t}+(\mathbf{v} \nabla) v_{i}\right]=0
$$

Here a new diffusional acceleration appears due to the presence of derivatives of the metrics in the Laplace-Beltrami operator $\nabla$

$$
E\left[\frac{\partial v_{i}}{\partial t}+(\mathbf{v} \nabla) v_{i}\right]=E\left[\Gamma_{i j}^{k} v^{j} v_{k}\right]
$$

The diffusional acceleration $E\left[\Gamma_{i j}^{k} j^{j} v_{k}\right]$ does not depend on the mass $m$ of the sample particle, i.e. we have an exact analog of the equivalence principle.

L. Smolin [4] had paid attention to the equality of the quantum diffusional mass $m_{q}$, determining from the diffusion coefficient $v=\hbar / 2 m_{q}$, and the inertial mass $m_{i n}$ with the high accuracy $\left(m_{i n}-m_{q}\right) / m_{i n}<4 \times 10^{-13}$. Here we can consider this fact as following from a generalized equivalence principle - the equivalence between the inertial motion in curved spacetime, the motion in a gravitational field and the inhomogeneous quantum diffusion.

Independence of the acceleration on the mass of the sample particle leads also to the same acceleration of macroscopic objects and the basises of local reference frames. The acceleration of a reference frame means the appearance of non-trivial macroscopic metric and non-zero curvature of space-time.

\section{The influence of matter to the stochastic background}

At the interaction with the stochastic background, a massive classical particle of a bare mass $m_{0}$ undergoes the stochastic fluctuations. At these fluctuations the energy of the vacuum around the particle partly transforms into the energy of particle's fluctuations. As the result, particle's energy increases to the quantum fluctuations energy, described by $T_{i k}^{(q)}\left(m_{0}^{-1}\right)$ which is inverse proportional to the bare mass $m_{0}$ and the physical energy-momentum density of matter becomes equal to: 


$$
T_{i k}(m)=T_{i k}^{(0)}\left(m_{0}\right)+T_{i k}^{(q)}\left(m_{0}^{-1}\right) .
$$

Due to this energy transfer the vacuum energy density around the source decreases with respect to the unperturbed vacuum at the spatial infinity. This lowering of the vacuum energy density is maximal near the source and vanishes at large distances. Such inhomogeneity of the vacuum energy density leads to a coordinate dependence of the diffusion coefficient $v_{i k}(x)$ which can be considered as the metric tensor of an effective Riemannian manifold. As it was shown in [1], the lowering of the energy density near the massive object leads to the diffusional acceleration of sample particles to that source so, that this acceleration does not depend on the mass of the sample particles.

As a physical model of this effect we can consider the behavior of two classical particles in an accelerated frame of reference. Let the first particle's trajectory be a geodesic line. This particle does not interact with the frame of reference and its "gravitational energy" is zero. Let the second particle be accelerated together with one of the local frames of the accelerated frame, and it is at rest in this frame. In this case the local frame expends the energy for the acceleration of the particle. As a result, the energy of the particle increases while the energy of the local frame interacting with the particle decreases. If the local frames at some surface of the extended reference frame are bonded by elastic springs (same as a trampoline), then the acceleration of the particle with one of the local frames leads to the formation of a smooth curved surface around this frame.

Thus, a gravitational energy density we can relate with the lowering of the vacuum energy density around the source, i.e. by the decreasing of the intensity of the quantum fluctuations. This fact we can take into account in the standard action function $A$ by the variation not the Lagrangian with the Ricci tensor $R_{i k}$, but directly containing the Riemann tensor $R_{i l k m}$ as in [6] (see earlier version in [ $\left.8^{*}\right]$ ):

$$
\begin{aligned}
A & =\frac{1}{2} \int d \Omega \sqrt{-\gamma}\left(-\kappa^{-1} R+L_{(m)}\right)= \\
& =\frac{1}{2} \int d \Omega \sqrt{-\gamma}\left[\frac{1}{2}\left(\gamma_{i l} \gamma_{k m}-\gamma_{i m} \gamma_{k l}\right)\left(-\kappa^{-1} R_{i k l m}+T_{i k l m}\right)+L_{(m)}\right] .
\end{aligned}
$$

Then we obtain a 4-index generalization of the Einstein equations:

$$
\begin{gathered}
\delta A=\frac{1}{2} \int d \Omega \sqrt{-\gamma}\left[-\kappa^{-1} G_{i k l m}+T_{i k l m}\right] \gamma^{i l} \delta \gamma^{k m}=0, \\
G_{i k l m}=\kappa T_{i k l m},
\end{gathered}
$$

which impose more strong constraints on observables of the gravitational field than the standard 2-index Einstein equations. Here:

$$
\begin{gathered}
G_{i k l m}=R_{i k l m}-\frac{1}{6}\left(\gamma_{i l} \gamma_{k m}-\gamma_{i m} \gamma_{k l}\right) R, \\
T_{i k l m}=V_{i k l m}+\frac{1}{2}\left(\gamma_{k m} T_{i l}-\gamma_{k l} T_{i m}+\gamma_{i l} T_{k m}-\gamma_{i m} T_{k l}\right)-\frac{1}{6}\left(\gamma_{i l} \gamma_{k m}-\gamma_{i m} \gamma_{k l}\right) T .
\end{gathered}
$$

The 4-index tensor of energy-momentum density of the source $T_{i k l m}$ contains the standard 2-index tensor of energy-momentum density of matter $T_{i k}$, its scalar $T=g^{i k} T_{i k}$ , and a new pure 4-index term $V_{i k l m}$ with zero contraction $g^{i l} V_{i k l m}=0$ which we will interpret the 4-index energy-momentum density tensor for the gravitational field. The 
latter follows from fact that in the vacuum $R_{i k l m}$ is equal to the Weyl tensor $C_{i k l m}$ which should be determined by the energy-momentum density of the gravitational field:

$$
C_{i k l m}=\kappa V_{i k l m} \text {. }
$$

In the asymptotically flat spacetime this definition of the gravitational energy leads to the same total energy of the source and its gravitational field as the pseudotensor and Hamiltonian approaches [6].

Another evidence of the quantum diffusional nature of gravitation is the explanation of the time dilation in the gravitational field. Due to the slowering of the quantum fluctuations around the massive source (the vacuum around had "lost" the energy for the fluctuating the source), the frequencies of the wave functions of the sample particles and energy levels of atoms, related with the intensity of the quantum fluctuations, become redshifted, i.e. all quantum processes near the source occur slower than at the spatial infinity.

\section{References}

1. Zakir Z. (2009) Theor. Phys., Astrophys. \& Cosmol., 4, No 1, 1; (1998) The theory of stochastic space-time. I. Gravitation as a quantum diffusion, arXiv:hep-th/9812254

2. Nelson E. (1966) Phys.Rev., 150, p. 1079.

3. Blanchard Ph., Combe Ph., Zheng W. (1987) Mathematical and Physical Aspects of Stochastic Mechanics, Lect.Not. in Phys., 281, 171 p.

4. Dohrn D., Guerra F. (1978) Lett. Nuovo Cim., 22, 4, p. 121.

5. Guerra F., Ruggiero P. (1978) Lett. Nuovo Cim., 23, 15, p. 529.

6. Smolin L. (1986) Phys.Lett., 113A, No 8, p. 408.

7. Zakir Z. (2009) Theor. Phys., Astrophys. \& Cosmol., 4, 2, 14; Four-index equations for gravitation and the gravitational energy-momentum tensor, arXiv:gr-qc/9906039.

8*. Gliner E., Dymnikova I. (1983) Phys. Rev. D28, 1278. 